\title{
Applications of Membrane Hydrocyclone Recovery of Silicon Carbide Powder
}

\author{
Yen-Cheng Tsai, Chen-Yang Hsu, Rome-Ming Wu* \\ Department of Chemical and Materials Engineering, Tamkang University, New Taipei City, Taiwan \\ Received 07 April 2019; received in revised form 07 May 2019; accepted 23 June 2019 \\ DOI: https://doi.org/10.46604/peti.2020.4205
}

\begin{abstract}
This work investigated the possibility of using membrane hydrocyclone as a separator to recover the silicon carbide powder. The weight percentage of silicon carbide powder is $62.9 \%$. Classifying these powders by a 2.5 $\mathrm{cm}$-diameter hydrocyclone that equips membrane tube in the center position, overflow and underflow are obtained. The results of the primary separation show that the overflow and underflow are about half of the proportion of solid, but silicon carbide powder content in the overflow is higher. Therefore, it is necessary to consider the recovery of silicon carbide powder contained in the overflow.
\end{abstract}

Keywords: separation, recovery, membrane, hydrocyclone, classification

\section{Introduction}

Today there is much-classifying equipment [1], and one of the most popular classifying equipment is hydrocyclone [2]. In the past, people made efforts to design new hydrocyclones with different structures in changing fluid characteristics and were committed to developing hydrocyclones with high separation efficiency. For example, assisted by electricity, [3] presented an electrical hydrocyclone with a central electrode rod. It's cut size decreases when the diameter and length of the electrode rod increase. [4] conceived of blocking the boundary layer flow, developing water injected hydrocyclone, so that small particles are no longer exist in the underflow. [5] put forward a filtering hydrocyclone which conical portion is replaced by the filter material, which enhances the classification efficiency. [6] put forward a hydrocyclone with an inlet, one underflow outlet, and two overflow outlets. The main functions of the two overflow outlets are to prevent large particles flow into the overflow by short-circuiting and to accelerate the prior separation of the particles in inner and outer overflow.

The velocity profiles of hydrocyclones were first measured by [7], he found that the tangential velocity increased to a maximum from hydrocyclone wall towards the center, and then decreased rapidly. Some studies agree on the same qualitative flow behavior [8-9]. Owing to a strong air core in the center, tangential and axial velocities become difficult to measure. However, with the development of science and technology, researchers focus on simulations by Computational Fluid Dynamics (CFD) techniques [10].

The performance of a hydrocyclone can be displayed in the following indexes:

(1) Flow split of a hydrocyclone is defined as the ratio of the volumetric flow rate of underflow to overflow.

(2) Separation efficiency Es:

$$
E_{s}=\frac{G_{u} c_{u}}{G_{f} c_{f}}
$$

\footnotetext{
* Corresponding author. E-mail address: romeman@mail.tku.edu.tw
}

Tel.: +886-2-26215656-3286; Fax: +886-2-26209887 
where $\mathrm{Gu}$ and $\mathrm{Gf}$ are mass flow rates of underflow and feed, respectively. cu and cf are weight concentrations of solids in underflow and feed, respectively.

(3) Reduced separation efficiency $E_{s}^{\prime}$

$$
E_{s}^{\prime}=\frac{E_{s}-R_{u f}}{1-R_{u f}}
$$

where Ruf is the ratio of volumetric flow rate of underflow to feed.

(4) Cut size $\mathrm{d}_{50}$ : The certain particle size that $50 \%$ particles flow out through underflow, and 50\% particles flow out through overflow.

(5) Partition curve: The probability that the particles with a certain size could be separated into underflow from the feed of hydrocyclone.

$$
P\left(d_{p}\right)=\frac{G_{u} c_{u}\left(d_{p}\right)}{G_{f} c_{f}\left(d_{p}\right)}
$$

Recently, [11] used CFD studies to simulate the separation of microorganisms and mammalian cells with hydrocyclones. Some works on the simulation of hydrocyclones using the incompressible Navier-Stokes equations, supplemented by a suitable turbulence model, have proven to be appropriate for modeling the flow in a hydrocyclone [12-13].

\section{Experimental Details}

\subsection{Materials}

Silicon carbide powder ranging from 2 23 $\mu \mathrm{m}$ diameter was used in this study. The particle size distribution was shown in Fig. 1. The average particle diameter is $16.5 \mu \mathrm{m}$, and the specific gravity of particles is 3.2. The adopted composite ceramic tubular membrane is made by TAMI. The support layer of the tubular membrane is made of Titania, the filter layer is made of Titania and Zirconia, with a pore size of $0.8 \mu \mathrm{m}$. The tubular membrane is with diameter $10 \mathrm{~mm}$, length $600 \mathrm{~mm}$, single channel, and a surface area of $0.008 \mathrm{~m}^{2}$. Laser particle size distribution analyzer, model No. Horrible LA-30 was used to measure particle size distribution from $0.1 \mu \mathrm{m} \sim 600 \mu \mathrm{m}$. By the light scattering theory, the light source with $405 \mathrm{~nm}$ of helium-neon LED was used to measure the sample particle size distribution on overflow and underflow.

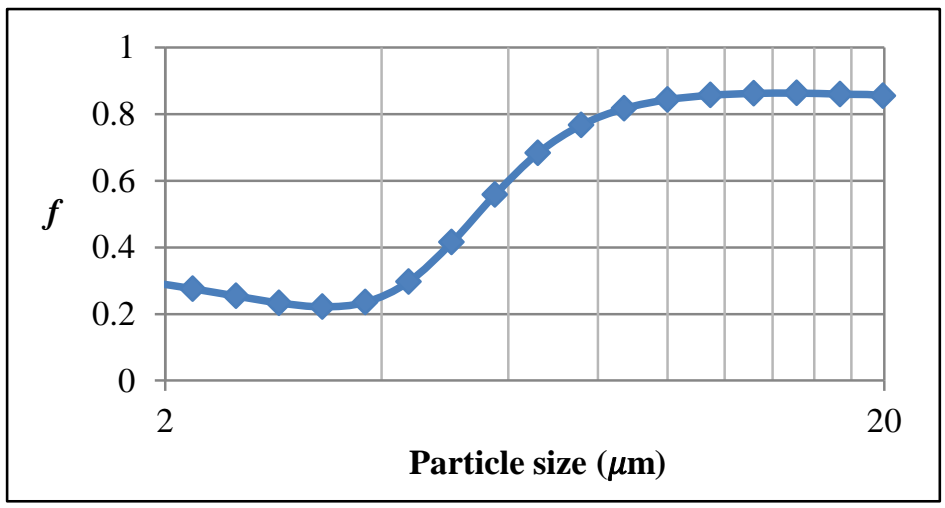

Fig. 1 PSD of silicon carbide powder

\subsection{Experimental setups and operation}

The geometry of the hydrocyclone is displayed in Fig. 2. The diameters of feed, overflow, underflow, and hydrocyclone are 5, 20, 15, and $25 \mathrm{~mm}$, respectively. The length of the cylindrical part is $40 \mathrm{~mm}$, while the length of the cone part is 110 $\mathrm{mm}$, making an overall $2.5^{\circ}$ cone angle. The experimental apparatus in this study is shown in Fig. 3. First, make sure the 
bypass valve (V3) is fully opened. Then fully open the overflow valve (V4) and underflow valve (V2), adjust the valve of feeding end (V1) and observe the feeding gauge pressure (P1) until the pressure is reached the one required. Record inlet pressure, the pressure at the overflow (P2) and the pressure at underflow (P3). Measure and sample the dilute liquid from overflow and the concentrated liquid stream from underflow, and record the amount of clear liquid filtrate at the outlet end of the outside-in filtration. Particles distribution, concentration, and separation efficiency were obtained.

\subsection{Simulation}

The simulation of the high-turbulent flow in hydrocyclone requires the basic equations of fluid dynamics combined with an adequate turbulence model. The flow pattern in hydrocyclone was modeled by Newtonian water flow and by Reynolds Stress Turbulence Model (RSM). The Reynolds-averaged Navier-Stokes equations are described as follows:

$$
\begin{aligned}
& \frac{\partial \rho}{\partial t}+\frac{\partial \rho}{\partial x_{i}}\left(\rho v_{i}\right)=0 \\
& \frac{\partial}{\partial t}\left(\rho v_{i}\right)+\frac{\partial}{\partial x_{j}}\left(\rho v_{i} v_{j}\right)=-\frac{\partial p}{\partial x_{i}}+\frac{\partial}{\partial x_{j}}\left[\mu\left(\frac{\partial v_{i}}{\partial x_{j}}+\frac{\partial v_{j}}{\partial x_{i}}-\frac{2}{3} \delta_{i j} \frac{\partial v_{j}}{\partial x_{j}}\right)\right]+\frac{\partial}{\partial x_{j}}\left(-\rho \overline{v_{i}^{\prime} v_{j}^{\prime}}\right)
\end{aligned}
$$

The exact transport equations for the transport of the Reynolds stresses, $\rho \overline{v_{i}^{\prime} v_{j}^{\prime}}$, may be written as follows:

$$
\begin{aligned}
\frac{\partial}{\partial t}\left(\rho \overline{v_{i}^{\prime} v_{j}^{\prime}}\right)+\frac{\partial}{\partial x_{k}}\left(\rho v_{k} \rho \overline{v_{i}^{\prime} v_{j}^{\prime}}\right)= & -\frac{\partial}{\partial x_{k}}\left[\rho \overline{v_{i}^{\prime} v_{j}^{\prime} v_{k}^{\prime}}+\overline{p\left(\delta_{k j} v_{i}^{\prime}+\delta_{i k} v_{j}^{\prime}\right)}\right]+\frac{\partial}{\partial x_{k}}\left[\mu \frac{\partial \overline{v_{i}^{\prime} v_{j}^{\prime}}}{\partial x_{k}}\right]-\rho\left(\overline{v_{i}^{\prime} v_{j}^{\prime}} \frac{\partial v_{j}}{\partial x_{k}}\right. \\
& \left.+\overline{v_{j}^{\prime} v_{k}^{\prime}} \frac{\partial v_{i}}{\partial x_{k}}\right)-\rho \beta\left(g_{i} \overline{v_{j}^{\prime} \theta}+g_{j} \overline{v_{i}^{\prime} \theta}\right)+p\left(\frac{\partial v_{i}^{\prime}}{\partial x_{j}}+\frac{\partial v_{j}^{\prime}}{\partial x_{i}}\right)-2 \mu \frac{\partial v_{i}^{\prime}}{\partial x_{k}} \frac{\partial v_{j}^{\prime}}{\partial x_{k}}
\end{aligned}
$$

where the left-hand side is local time derivative and convection terms, The right-hand side are turbulent diffusion, molecular diffusion, stress production, buoyancy production, pressure strain, and dissipation terms, respectively.

A velocity inlet boundary condition was applied at the inlet:

$$
\vec{u}=\text { const. @ inlet pipe }
$$

The overflow and underflow used pressure outlet boundary conditions. The outlet fluid is moving under absolute pressure $1 \mathrm{~atm}$ therefore the gauge pressure at the overflow and underflow are zero. The boundary conditions are:

$$
\begin{array}{ll}
P=0 & @ \text { underflow } \\
P=0 & @ \text { overflow }
\end{array}
$$

The computational fluid dynamics program FLUENT 6.2 (Fluent Inc., USA) solved the governing equations, Eqs. (4-7), together with the associated boundary conditions Eqs. (7-9). Simulations were carried out for about 50,000 incremental steps where in general a preset value of convergence criteria $1 \times 10^{-4}$ was achieved. FLUENT predicts the trajectory of a discrete phase particle by integrating the force balance on the particle. This force balance can be written as (for $x$ direction):

$$
\begin{aligned}
& \frac{d u_{p}}{d t}=F_{D}\left(u-u_{p}\right)+\frac{g_{x}\left(\rho_{p}-\rho\right)}{\rho_{p}} \\
& F_{D}=\frac{18 \mu}{\rho_{p} d_{p}^{2}} \frac{C_{D} \operatorname{Re}}{24}
\end{aligned}
$$

where $F_{D}\left(u-u_{p}\right)$ is the drag force per unit particle mass. 
where $u$ is the fluid phase velocity, $u_{p}$ is the particle velocity, $\mu$ is the molecular viscosity of the fluid, $\rho$ is the fluid density, $\rho_{p}$ is the density of the particle, and $d_{p}$ is the particle diameter. Re is the relative Reynolds number, which is defined as

$$
\operatorname{Re}=\frac{\rho d_{p}\left|u_{p}-u\right|}{\mu}
$$

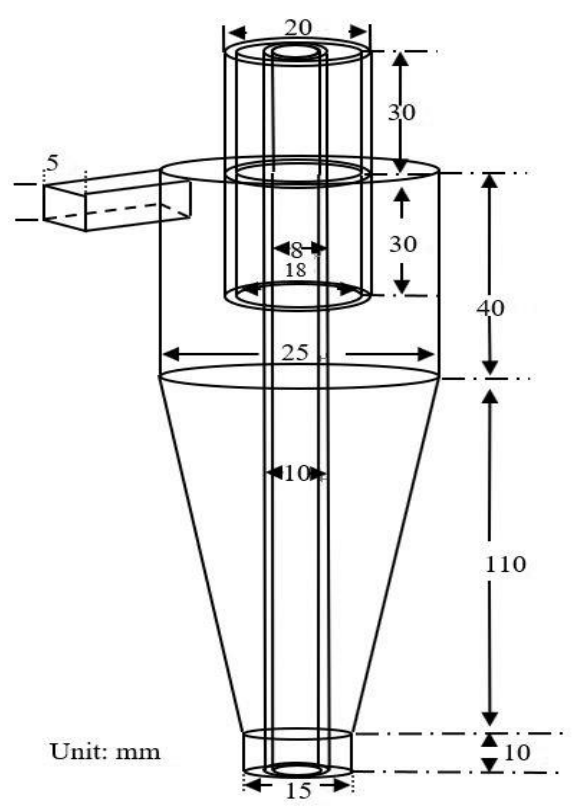

Fig. 2 Geometry of membrane hydrocyclone

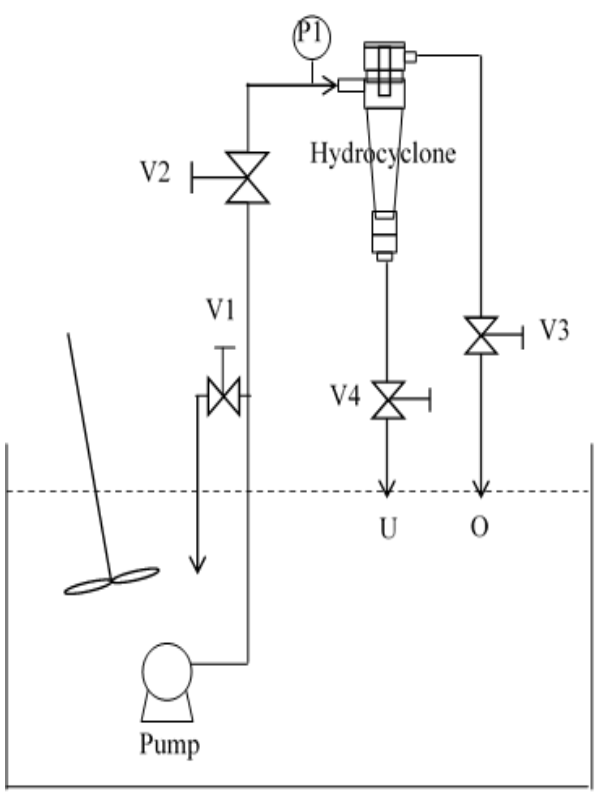

Fig. 3 Creative design methodology

\section{Result}

The meaning of the classification efficiency curve is the rate of a particle of a certain size flowing from the feeding inlet of the hydrocyclone and leaving from the underflow of the hydrocyclone. Figure 4 is the velocity distribution under different operating pressures. Fig. 5 is the classification efficiency curve of the separators under the same feeding conditions.

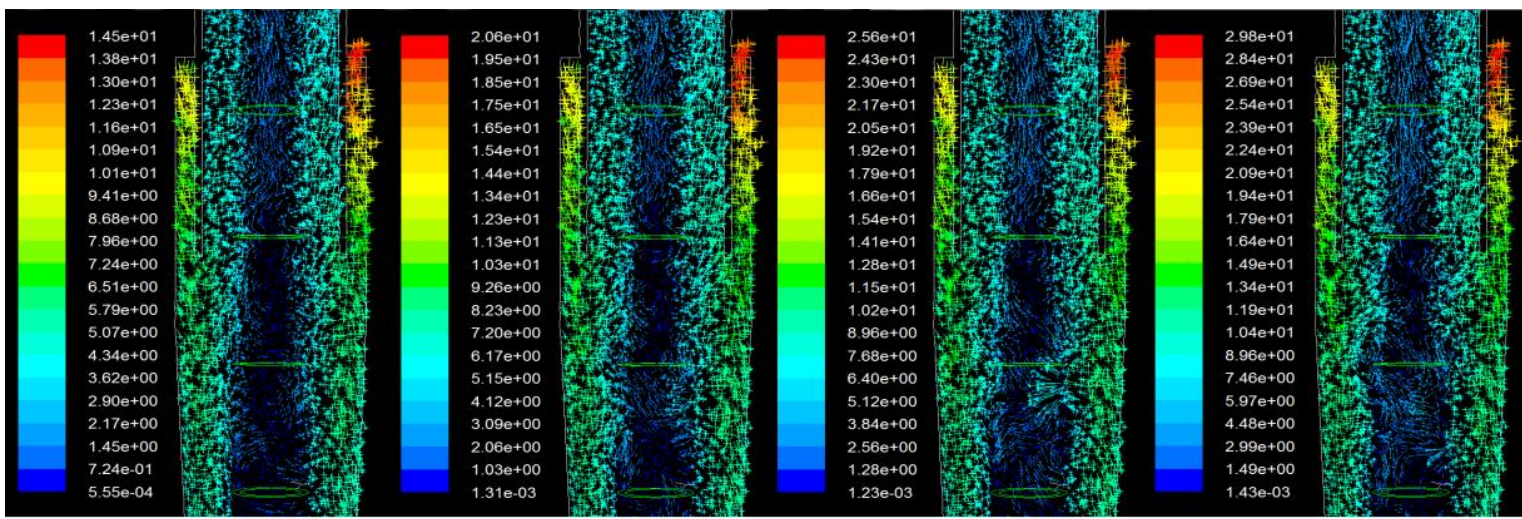

Fig. 4 Velocity distribution for 0.5 bar, 1.0 bar, 1.5 bar, and 2.0 bar

The separation efficiency is enhanced by a series of hydrocyclone separator in order to recover silicon carbide of higher purity. The separation system does not have to completely replace the membrane hydrocyclone with a conventional separator, but rather consider its application location. Finally, the membrane hydrocyclone in order to enhance the filtrate production to show its characteristics, need to explore the parameters of the experiment, such as raising the inlet pressure, change the membrane tube, adjust the concentration of the liquid and temperature.

Although the overall separation efficiency is slightly less than tube without membrane, the single membrane hydrocyclone has a good impact on separation efficiency. 
A dual feed hydrocyclones is proposed for separating an aqueous slurry of particles into a top stream which contains the light particles and a bottom stream which contains large particles [18]. A silicon containing material can be processed for production of a silicon-rich composition and can be used to form a wafer-saw cutting fluid [19]. The invention of hydrocyclone system for recovering silicon and silicon-containing compounds from slurry that is generated during wafer cutting or sawing operations in the Photovoltaic (PV) and Microelectronic (ME) industries. The system can recover at least $60 \%$ of the silicon contained in a silicon-cutting slurry. A study on recycling silicon and silicon carbide powders from wiresaw slurry containing silicon carbide, silicon kerfs, Polyethylene Glycol (PEG), and iron fragments was conducted [20]. After the first stage treatment of silicon carbide powders on hydrocyclones, $85 \mathrm{wt} \%$ silicon carbide powders can be collected at underflow. In this study, the weight percentage of original silicon carbide powder is $62.9 \%$. After separation, it can concentrate to $83.4 \mathrm{wt} \%$. In addition, it can get $38.2 \mathrm{~L} / \mathrm{hr}-\mathrm{m} 2$ clear PEG filtrate.

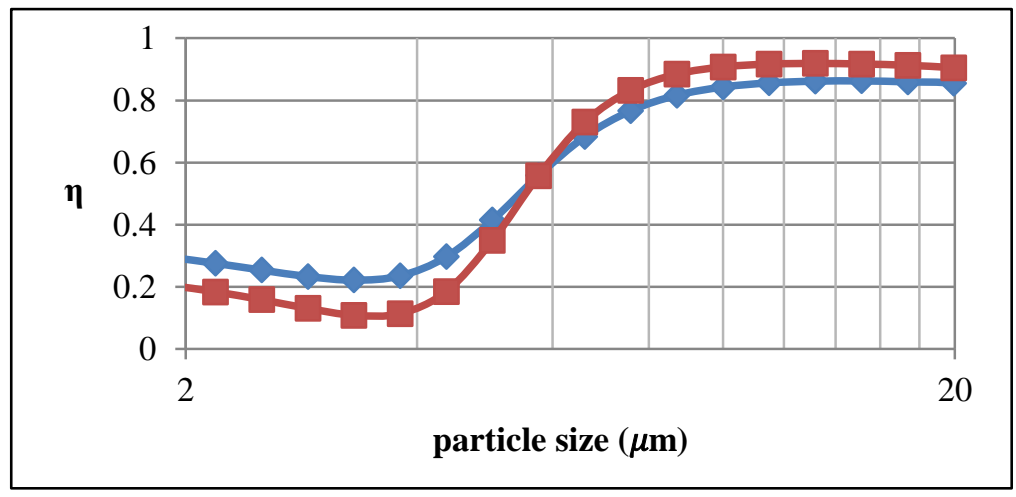

Fig. 5 Plots of separation efficiency versus particle size on different operations (Blue curve: equips membrane tube; Red curve: tube without membrane)

A dual feed hydrocyclones is proposed for separating an aqueous slurry of particles into a top stream which contains the light particles and a bottom stream which contains large particles [18]. Silicon-containing material can be processed for the production of a silicon-rich composition and can be used to form a wafer-saw cutting fluid [19]. The invention of hydrocyclone system for recovering silicon and silicon-containing compounds from the slurry that is generated during wafer cutting or sawing operations in the Photovoltaic (PV) and Microelectronic (ME) industries. The system can recover at least $60 \%$ of the silicon contained in a silicon-cutting slurry. A study on recycling silicon and silicon carbide powders from wiresaw slurry containing silicon carbide, silicon kerfs, Polyethylene Glycol (PEG), and iron fragments was conducted [20]. After the first stage treatment of silicon carbide powders on hydrocyclones, $85 \mathrm{wt} \%$ silicon carbide powders can be collected at underflow. In this study, the weight percentage of original silicon carbide powder is $62.9 \%$. After separation, it can concentrate on $83.4 \mathrm{wt} \%$. In addition, it can get $38.2 \mathrm{~L} / \mathrm{hr}-\mathrm{m}^{2}$ clear PEG filtrate.

\section{Conclusions}

In this study, silicon carbide particles were used to implement hydrocyclone experiments. The air core area which is replaced by ceramic tubular membrane was discussed, and the effects of separation phenomena of different hydrocyclones were explored. By inserting a tubular membrane, the hydrocyclone becomes a new-type hydrocyclone with three outlets. They are respectively the dilute liquid of overflow, the concentrated liquid of underflow, and the clear filtrate. It is as well equipped with both functions of classification and filtration. Comparing with conventional hydrocyclone, it has equivalent classification function, but the overflow concentration is relatively dilute, the underflow concentration is relatively concentrated, and clear filtrate can be obtained at the same time.

\section{Conflicts of Interest}

The authors declare no conflict of interest. 


\section{Acknowledgment}

The authors would like to thank the National Science Council of the Republic of China, Taiwan, for financially supporting this research under contract No. NSC 97-2221-E-032-030-MY3.

\section{References}

[1] J. Margraf and J. Werther, "Continuous classification in a centrifugal fluidized bed apparatus," Journal of the Taiwan Institute of Chemical Engineers, vol. 40, pp. 669-681, 2009.

[2] K. Nageswararao, "A critical analysis of the fish hook effect in hydrocyclone classifiers," Chemical Engineering Journal, vol. 80, pp. 251-256, 2000.

[3] H. Yoshida, S. Taniguchi, and K. Fukui, "Effect of apex cone on particle classification performance of cyclone separator, Journal of the Taiwan Institute of Chemical Engineers, vol. 35, pp. 41-46, 2004.

[4] D. D. Patil and T. C. Rao, "Classification evaluation of water injected hydrocyclone," Minerals Engineering, vol. 12, pp. 1527-1532, 1999.

[5] L. G. M. Vieira, E. A. Barbosa, J. J. R. Damasceno, and M. A. S. Barrozo, "Performance analysis and design of filtering hydrocyclones," Brazilian Journal of Chemical Engineering, vol. 22, pp. 143-152, 2005.

[6] A. Mainza, M. S. Powell, and B. Knopjes, "Differential classification of dense material in a three-product cyclone," Minerals Engineering, vol. 17, pp. 573-579, 2004.

[7] D. F. Kelsall, "A Study of the Motion of Solid Particles in a hydraulic cyclone,” Trans Instn Chem Engrs., vol. 30, pp. 87-108, 1952.

[8] J. Bergstrom and H. Vomhoff, "Experimental hydrocyclone flow field studies," Separation and Purification Technology, vol. 53, pp. 8-20, 2007.

[9] J. Bergstrom, H. Vomhoff, and D. Soderberg, "Tangential velocity measurements in a conical hydrocyclone operated with a fibre suspension,” Minerals Engineering, vol. 20, pp. 407-413, 2007.

[10] C. Y. Hsu and R. M. Wu, "Hot zone in a hydrocyclone for particles escape from overflow," Drying Technology, vol. 26, pp. 1011-1017, 2008.

[11] R. A. Medronho, J. Schuetze, and W. D. Deckwer, "Numerical simulation of hydrocyclones for cell separation," Latin American Applied Research, vol. 35, pp. 1-8, 2005.

[12] B. Wang, K. W. Chu, and A. B. Yu, "Numerical study of particle-fluid flow in a hydrocyclone," Ind Eng Chem Res., vol. 46, pp. 4695-4705, 2007.

[13] B. Wang and A. B. Yu, "Numerical study of the gas-liquid-solid flow in hdrocyclones with different configuration of vortex finder," Chemical Engineering Journal, vol. 135, pp. 33-42, 2008.

[14] L. Y. Chu, W. M. Chen, and X. Z. Lee, "Enhancement of hydrocyclone performance by controlling the inside turbulence structure," Chemical Engineering Science, vol. 57, pp. 207-212, 2002.

[15] B. Li, M. Huang, T. Fu, L. Pan, W. Yao, and L. Guo, "Microfiltration process by inorganic membranes for clarification of TongBi liquor,” Molecules, vol. 17, pp. 1319-1334, 2012.

[16] M. Kim, J. H. Kim, A. S. Syed, Y. M. Kim, K. K. Choe, and C. Y. Kim, “Application of centrifugal partition chromatography for bioactivity-guided purification of antioxidant-response-element-inducing constituents from atractylodis rhizoma alba,” Molecules, vol. 23, pp. 2274-2283, 2018.

[17] R. M. Wu, "Hydrocyclone separator," US Patent, US 8182684 B1, May 2012.

[18] F. G. Miller, "Dual feed hydrocyclone and method of separating aqueous slurry," US Patent, US 4652363, March 1987.

[19] J. A. Fallavollita, "Methods and apparatus for recovery of silicon and silicon carbide from the spent wafer-sawing slurry," US Patent, US 2011/0059002 A1, March 2011.

[20] S. A. Sergiienko, B. V. Pogorelov, and V. B. Daniliuk, "Silicon and silicon carbide powders recycling technology from wire-saw cutting waste in slicing process of silicon ingots," Separation and Purification Technology, vol. 133, pp. 16-21, 2014.

Copyright $($ by the authors. Licensee TAETI, Taiwan. This article is an open access article distributed under the terms and conditions of the Creative Commons Attribution (CC BY-NC) license (https://creativecommons.org/licenses/by-nc/4.0/). 